\title{
Atopic Dermatitis Oral Therapies: What Are Patients Learning on YouTube?
}

\author{
Sheida Naderi-Azad, MD; Mirjana G. Ivanic, BSc; Shikha Walia, BSc; Jashin J. Wu, MD
}

\section{PRACTICE POINTS}

- Patient-based YouTube videos comprised the majority of videos on oral therapies for atopic dermatitis, with the greatest views and interaction ratio.

- Most YouTube videos on this topic contained a neutral tone and Grade B recommendations, thus meriting production of more evidence-based videos in collaboration with patients on the YouTube platform.

To the Editor:

Oral immunosuppressive therapies are prescribed for moderate to severe atopic dermatitis. Patients often consult YouTube to make informed decisions about these therapies. In the United States, most health-related online searches are initiated through a search engine, which frequently leads to social media sites such as YouTube. Recent studies have examined the reasons why users turn to the Internet for health-related information, indicating that users typically seek specific information regarding health concerns. ${ }^{1,2}$ Furthermore, social media platforms such as YouTube are a popular means of sharing health information with the public..$^{3-5}$ Currently, YouTube has more than 1 billion registered users, and 30 million health-related videos are watched each day. ${ }^{6}$ Almost onethird of US consumers use YouTube, Facebook, and Twitter to obtain medical information. ${ }^{7}$ YouTube is a versatile tool because of its video-discovery mechanisms such as a keyword-based search engine, video-recommendation system, highlight feature for videos on home pages, and the capacity to embed YouTube videos on various web pages. ${ }^{8}$ Searchers use videos that are short, fast paced, emotion evoking, from credible sources, recently uploaded, and relevant to the searcher for aiding in health decisions. ${ }^{9}$ Furthermore, studies have demonstrated YouTube's capacity to support a change in attitude and increase users' knowledge. In fact, YouTube had higher impact on recall, attitudes, and behaviors when compared with written materials on other social media platforms, such as Facebook and Twitter. ${ }^{9}$ We conducted a crosssectional study to examine the quality of YouTube videos on oral therapies for atopic dermatitis, such as cyclosporine, methotrexate, azathioprine, and mycophenolate mofetil.

On April 23, 2020, we performed 8 searches using a private browser with default filters on YouTube (Figure). Injectables were not included in the analysis, as the YouTube experience on dupilumab previously has been investigated. ${ }^{10}$ The top 40 videos from each search were screened by 3 researchers. Duplicates, non-Englishlanguage videos, and videos that did not discuss atopic dermatitis or oral therapies were excluded, resulting in 73 videos included in this analysis. Testimonials generated by patients made up 39 of 73 (53.4\%) videos. Health care professionals created 23 of $73(31.5 \%)$ videos, and educators with financial interest created 11 of 73 (15.1\%) videos. The dates of production for the videos spanned from 2008 to 2020.

The major topics addressed in the videos were symptomatic changes (63 [68.8\% of all topics discussed]), adverse effects (52 [67.5\%]), and quality-of-life changes (37 [48.1\%]). Of the videos included, the majority (42/73 [57.5\%]) contained a neutral tone about the medication, citing advantages and disadvantages with therapy, while 22 of $73(30.1 \%)$ had an encouraging tone, and 9 of $73(12.3 \%)$ had a discouraging tone. Regarding videos with positive tones, there were 17 videos on

Dr. Naderi-Azad is from the University of Toronto, Ontario, Canada. Ms. Ivanic is from Meharry Medical College, Nashville, Tennessee. Ms. Walia is from Lake Erie College of Osteopathic Medicine, Bradenton, Florida. Dr. Wu is from Dermatology Research and Education Foundation, Irvine, California. Ms. Naderi-Azad, Ms. Ivanic, and Ms. Walia report no conflict of interest. Dr. Wu is or has been an investigator, consultant, or speaker for AbbVie; Almirall; Amgen; Arcutis Biotherapeutics; Boehringer Ingelheim; Bristol-Myers Squibb; Celgene; Dermavant Sciences, Inc; Dermira, Inc;

Dr. Reddy's Laboratories; Eli Lilly and Company; Janssen Pharmaceuticals; LEO Pharma; Novartis; Regeneron Pharmaceuticals; Sanofi Genzyme; Sun Pharmaceutical Industries Ltd; UCB; and Valeant Pharmaceuticals North America LLC.

Correspondence: Jashin J. Wu, MD (jashinwu@hotmail.com).

doi:10.12788/cutis.0346 


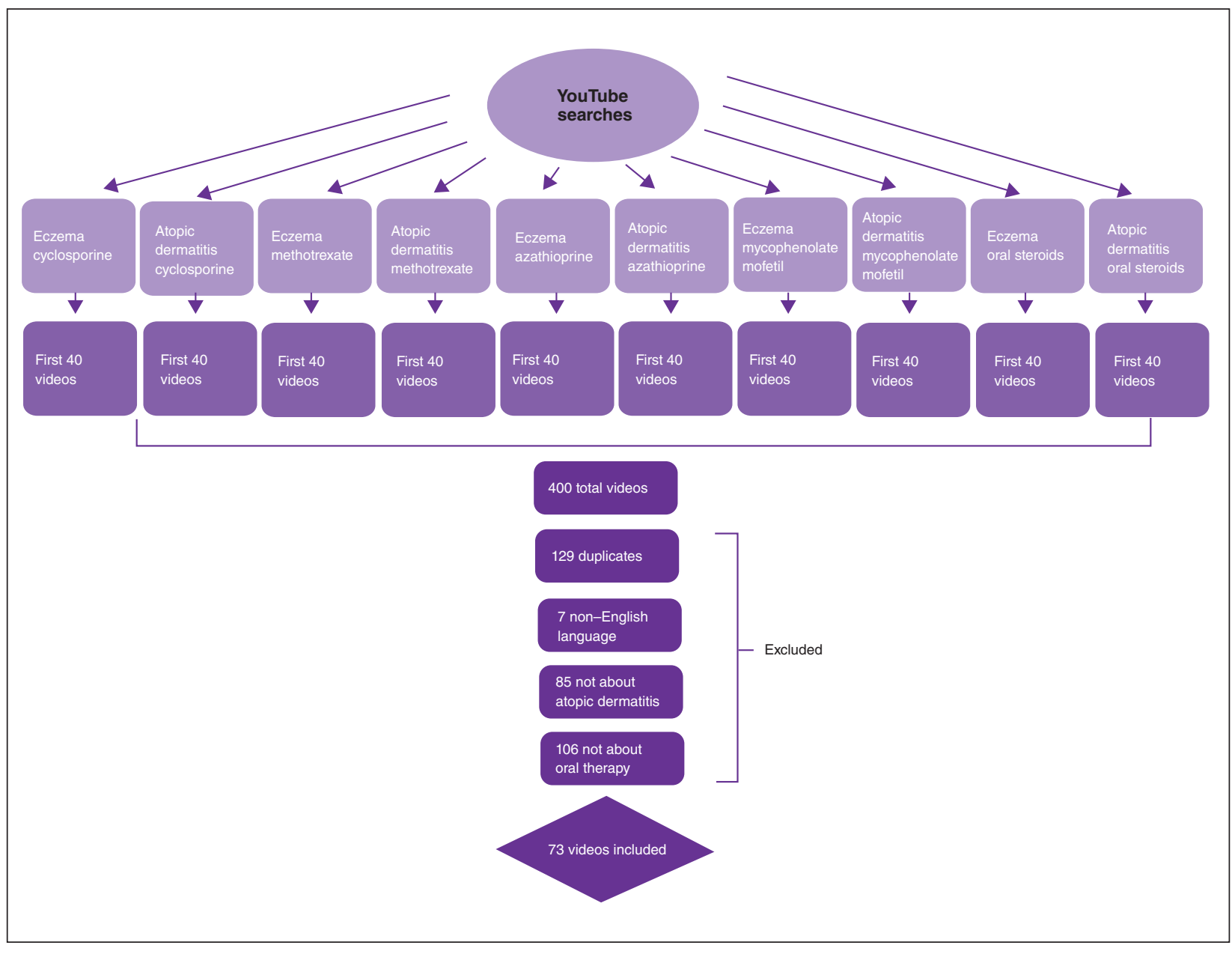

Algorithm for YouTube searches on oral therapies for atopic dermatitis and process of video exclusion.

cyclosporine, 9 on azathioprine, 7 on methotrexate, 4 on oral steroids, and 2 on mycophenolate mofetil. Regarding videos with negative tones, there were 4 on cyclosporine, 3 on azathioprine, 2 on methotrexate, and 2 on mycophenolate mofetil.

Of the videos made with financial interest, the majority (28/34 [77.8\%]) were more suitable for informing health care providers rather than patients, containing jargon as well as complex information on clinical trials, dosing, and mechanisms of action. From the videos discussing clinical recommendations, there were 9 of 73 (12.3\%) Grade A recommendations (eg, citing evidence-based information and clinical trials) and 64 of $73(87.7 \%)$ Grade B recommendations (eg, anecdotal information on patient experience). Thirty-seven of 73 (50.7\%) videos were evidence based, and 36 of $73(49.3 \%)$ were non-evidence based. Six videos were patient-oriented news broadcasts.

Patient-generated testimonials had the most views (mean, 9238.4) and highest interaction ratio (the sum of likes, dislikes, and comments divided by the number of views)(mean, 0.027), while health care providergenerated videos had fewer views (mean, 9218.7) and a lower interaction ratio (mean, 0.011). Financial-based videos had 4233.4 views on average, with an average interaction ratio of 0.014 . Based on these results, biased, patient-generated content comprised greater than 50\% of YouTube videos about oral therapies for atopic dermatitis and was quite likely to be engaged with by users. Thus, these patient testimonials have great potential to affect decision-making.

The high number of patient-generated videos about oral therapies was consistent with prior studies of YouTube videos about therapies for numerous conditions. ${ }^{11-13}$ Dermatologists should consider utilizing YouTube for providing evidence-based, patient-oriented information about novel therapeutics. They may consider collaborating with patients to assist with their creation of YouTube videos and directing patients to credible resources by the American Academy of Dermatology and Canadian Dermatology Association for decision-making.

Importantly, this analysis is limited by its lack of quality-assessment tools for video-based resources such as JAMA score and DISCERN score. ${ }^{14,15}$ However, CONTINUED ON PAGE 157 
CONTINUED FROM PAGE 154

these metrics have limited ability to evaluate audiovisual elements, indicating the need for novel tools to score their validity.

\section{REFERENCES}

1. Fox S, Duggan M. Health online 2013. January 15, 2013. Accessed August 15, 2021. https://www.pewresearch.org/internet/2013/01/15 /health-online-2013/

2. Ní Ríordáin R, McCreary C. Dental patients' use of the Internet. Br Dent J. 2009;207:583-586, 575.

3. Fergie G, Hilton S, Hunt K. Young adults' experiences of seeking online information about diabetes and mental health in the age of social media. Health Expect. 2016;19:1324-1335.

4. Antheunis ML, Tates K, Nieboer TE. Patients' and health professionals' use of social media in health care: motives, barriers and expectations. Patient Educ Couns. 2013;92:426-431.

5. McGregor F, Somner JE, Bourne RR, et al. Social media use by patients with glaucoma: what can we learn? Ophthalmic Physiol Opt. 2014;34:46-52.

6. YouTube Statistics-15 Amazing Stats for 2015. Published April 30, 2015. Accessed August 27, 2021. YouTube.com /watch?v=9ZLBSPzY7GQ

7. Health Research Institute. Social media "likes" healthcare: from marketing to social business. April 2012. Accessed August 15, 2021. https://www.pwc.com/us/en/health-industries/health-research -institute/publications/pdf/health-care-social-media-report.pdf

8. Zhou R, Khemmarat S, Gao L, et al. How YouTube videos are discovered and its impact on videos views. Multimed Tools Appl. 2016;75:6035-6058.

9. Haslam K, Doucette H, Hachey S, et al. YouTube videos as health decision aids for the public: an integrative review. Can J Dent Hyg. 2019;53:53-66.

10. Pithadia D, Reynolds K, Lee E, et al. Dupilumab for atopic dermatitis: what are patients learning on YouTube [published online ahead of print April 16,2020]? J Dermatolog Treat. doi: 10.1080/09546634.2020.1755418

11. Tolu S, Yurdakul OV, Basaran B, et al. English-language videos on YouTube as a source of information on self-administer subcutaneous anti-tumour necrosis factor agent injections. Rheumatol Int. 2018;38:1285-1292.

12. Reynolds KA, Pithadia DJ, Lee EB, et al. A cross-sectional study of YouTube videos about psoriasis biologics. Int J Dermatol. 2019; 58:E61-E62.

13. Kocyigit BF, Akaltun MS. Does YouTube provide high quality information? assessment of secukinumab videos. Rheumatol Int. 2019;39:1263-1268.

14. Qi J, Trang T, Doong J, et al. Misinformation is prevalent in psoriasisrelated YouTube videos. Dermatol Online J. 2016;22:13030/qt7qc9z2m5

15. Gokcen HB, Gumussuyu G. A quality analysis of disc herniation videos on YouTube. World Neurosurg. 2019;124:E799-E804. 